\title{
Organic material in meteorites and the link to the origin of life
}

\section{Remusat ${ }^{1}$}

${ }^{1}$ Laboratoire de Minéralogie et Cosmochimie du Museum, UMR CNRS 7202, CP52 57 rue Cuvier, 75231 Paris Cedex 05, France (remusat@mnhn.fr)

\begin{abstract}
Life requires specific conditions that have been, so far, only proven to meet on Earth. Though the chemical elements required to form living organism $(\mathrm{C}, \mathrm{H}, \mathrm{N}, \mathrm{O}$, $\mathrm{S}$, etc) are widespread in the universe, the molecules that are crucial for Life, like nucleobases or amino acids, may not be so ubiquitous. The question of the formation of small and complex molecules is highly relevant to understand the process of Life origin. Carbonaceous chondrites are a class of meteorites rich in organic compounds and host potential precursors for the emergence of Life (organic matter and water). They could have been the source of complex molecules on the early Earth. This contribution will describe the main properties of the organic matter recovered from carbonaceous chondrites. However, the isotopic and molecular record of organic compounds is faded by secondary processes that occurred on the parent body of these meteorites. This results in complex signatures that raise multiple questions about the origin of organic compounds in the Solar System.
\end{abstract}

\section{Introduction}

The Solar System formed from a dusty molecular cloud 4.56 billion years ago. During its first ages, the condensation of materials resulted in the formation of solids that hereafter accreted to form planet embryos. These solids have accreted ices, organic grains and silicate particles recording the signature of the processes occurring during the birth of the Solar System. Chondrites are undifferentiated meteorites and are considered as primitive objects in the solar system, along with micrometeorites, interplanetary dust particles (IDPs) and cometary grains. They result from the disruption of small bodies that did not undergo differentiation, i.e. internal heating was not enough to induce the formation of layers with different mineralogical and chemical composition by melting/crystallization in contrast to terrestrial planets, like the Earth.

Several classes of chondrites are defined depending on their chemical and mineralogical composition. Carbonaceous chondrites are rich in carbon and organic matter, and they contain a large amount of volatile elements (with condensation temperatures below 640K) like $\mathrm{H}$, noble gases, halogens, $\mathrm{Pb}$ or $\mathrm{Hg}$ [1]. They are the oldest meteorites in the solar system [2] and their elemental composition is close to the composition of the Sun, except for the most volatile elements like $\mathrm{H}, \mathrm{He}$, $\mathrm{C}, \mathrm{O}$ and $\mathrm{N}$ [3]. They are considered to reflect the composition of the molecular cloud that gave birth to the solar system [4]. 
Carbonaceous chondrites contain up to 4 wt.\% of organic matter occurring as soluble compounds and macromolecular insoluble organic matter (IOM). It is found in the matrix of chondrites, associated with phyllosilicates [5]. Organics likely accreted with other volatiles components in the matrix. This is confirmed by fluorescence microscopy [6], which shows that organic matter occurs as circular particles, evenly distributed in the matrix of hydrated chondrites and with a diameter around a couple of microns. Organic matter has also been observed to form nanoglobules [7, 8]; analogues were produced during laboratory experiment intending to reproduce ejecta gas from evolved stars [9].

However, some chemical reactions have occurred since the chondrites accretion. The chondrites parent bodies (i.e. the parent asteroids) accreted significant amount of short live radionucleides, like for instance ${ }^{26} \mathrm{Al}$. The decay of these isotopes has resulted in some modifications of the accreted components by hydrothermal (aqueous) alteration or thermal metamorphism on the parent body [10, 11]. Extends of these modifications are variable among chondrites class depending on the size/composition of their parent body $[12,13]$. CI are the most hydrated carbonaceous chondrites, $\mathrm{CM}$ are less hydrated. CR are often considered as the least modified carbonaceous chondrites on their parent bodies, with the less processed materials. They are supposed to have been subjected to mild metamorphism and hydrothermalism. $\mathrm{CO}$ and $\mathrm{CV}$ are the metamorphosed counter parts of the $\mathrm{CM}$. CO and CV differ by their content in refractory elements and the size of their chondrules (both being larger in $\mathrm{CV}$ ). They have been subject to temperatures higher than $350^{\circ} \mathrm{C}$, and contain much less organics and water [14].

\section{Diversity of organic compounds in meteorites}

Organic matter in carbonaceous chondrites can be spread in an insoluble macromolecule [15] and a complex suite of soluble organic molecules $[16,17]$. The total organic content may reach $4 \mathrm{wt} . \%$ in some chondrites, with the macromolecular carbon representing more than $75 \mathrm{wt} \%$. As recently shown [18], the soluble organic content in carbonaceous chondrites is very diverse. The use of very high mass resolution techniques revealed the occurrence of 14,000 different molecules, containing C, $\mathrm{H}, \mathrm{O}, \mathrm{N}$ and $\mathrm{S}$. These molecules can be divided into several classes (see figure 1), including sugar derivatives [19], amino acids [17] and nucleobases [20] being potential metabolic reactants and genetic information recorders. The most abundant soluble compounds in Murchison are carboxylic acids (330 ppm [16]). These soluble compounds share several molecular properties [21]: 1- they show a complete structural diversity (for each formula, every isomer is detected), 2- the abundance decreases when the carbon number increases and in general 3- branched chains are more abundant.

The soluble compounds exhibit a complex isotopic distribution pointing to a complex origin [22]. The soluble fraction is likely a mixing between moieties that could have been formed on the parent body, from the reaction of precursors that could have been accreted in ices or from the hydrolysis of the IOM, and compounds that could have been synthesized prior to accretion and preserved trapped in the matrix of the carbonaceous chondrites. The question of the genetic link between soluble and insoluble organic matter is still debated; the IOM could for instance release some soluble compounds during hydrothermal alteration [23, 24]. To date, only aromatic hydrocarbons could be related to the macromolecular carbon [23].

Amino acids are emblematic organic compounds in meteorites. They are often cited as possible prebiotic molecules for the origin of life and are known to be indigenous in meteorites since the 70's $[17,25]$. To date, about 80 amino acids have been detected in CI, CM and CR chondrites; many of them have no terrestrial counterparts [26]. Their abundance varies between meteorites from less than a few ppm in some CI up to $250 \mathrm{ppm}$ in some CR2 [27]. Amino acids in meteorites have from 2 to 8 carbon atoms; all the possible isomers are present. The distribution of amino acids differs from one meteorite to the other. For instance, in Murchison, glycine $>\alpha$-amino-isobutyric acid $>$ alanine, whereas in Orgueil $\beta$-alanine $>$ glycine $>\gamma$-amino-butyric acid. Branched chains are more abundant than straight ones. Moreover, $\alpha, \beta$ and $\gamma$ amino acids can be detected, with an abundance order $\alpha>\gamma>$ $\beta$. Molecular and isotopic data on extraterrestrial amino acids indicate a complex origin for this 
fraction [29]: the synthetic pathway and evolution depends on the parent body. So far there is no consensus about the processes ruling the distribution of amino acids or their isotopic compositions. The correlation between the distribution of amino acids and hydroxy acids has lead to the suggestion that amino acids were synthesized by Strecker-cyanohydrin synthesis [28]. Indeed, this reaction can form, from aldehydes or ketone precursors, $\alpha$-amino acids and $\alpha$-hydroxy acids that will have parallel distribution. This reaction requires $\mathrm{HCN}$ and water as co-reactants. But this reaction cannot produce the non $\alpha$-amino acids and other mechanisms are required [29].

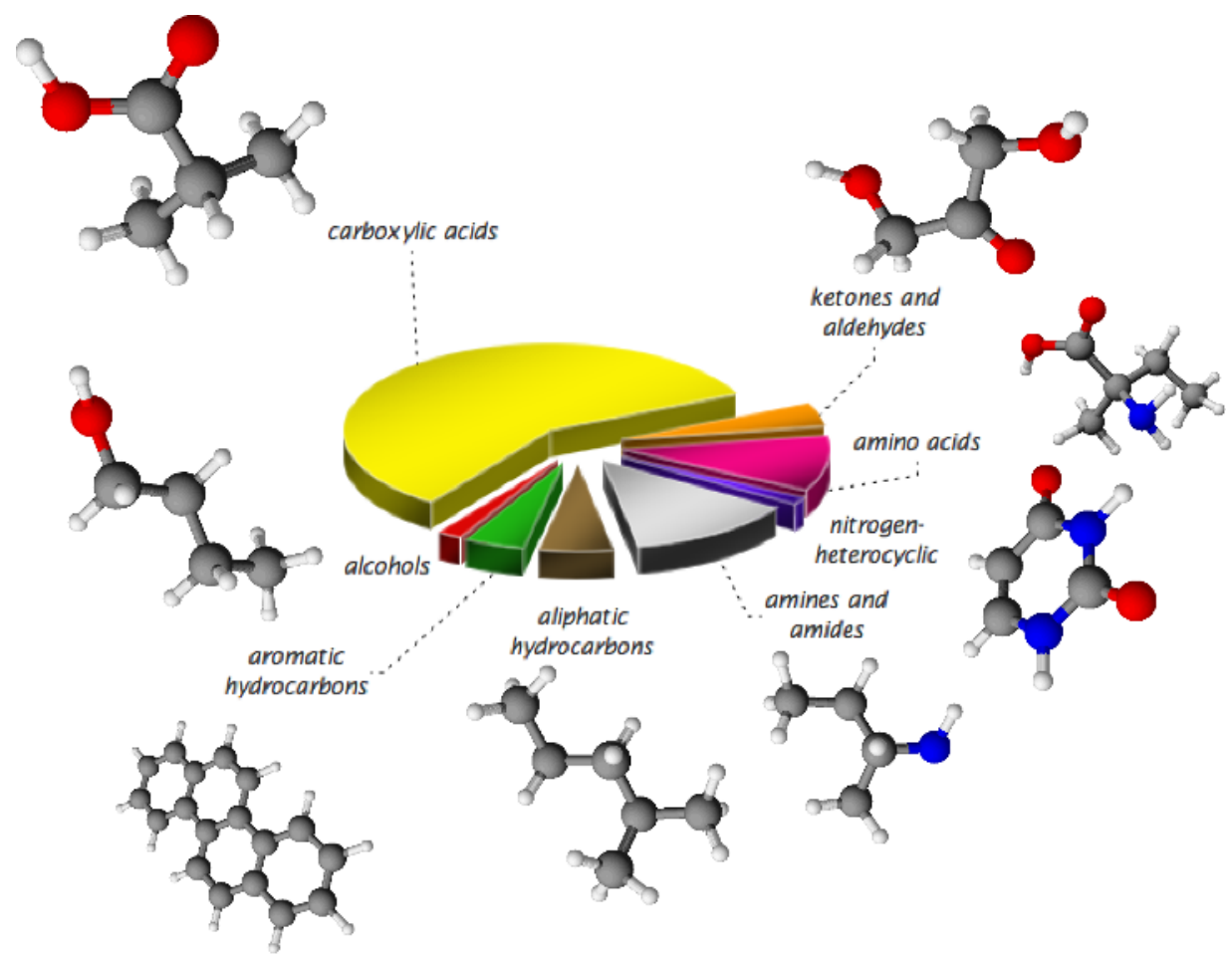

Fig. 1. Distribution and molecular models of soluble molecules in the Murchison meteorite.

Enantiomeric excesses have been detected in chondrites, in amino acids [30, 31]. Enantiomeric excesses are variable among meteorites [31], and also inside the same objects [32] but do not exceed $18 \%$. The hydrated meteorites exhibit higher excess, pointing to a possible amplification during aqueous alteration [31]. The exact process by which this amplification would occur is unknown, but some chemical reactions, like the Soai reaction [33] exhibit the property to enhance enantiomeric excess. Effect of UV circularly polarized light in space (gas phase or ices) has been suggested as an alternative way to induce excesses observed [34]. This hypothesis is being tested by laboratory experiments [35-37] but the source of the CPL at the vicinity of the early solar system still needs to be assessed. Chirality has been suggested to also occur in the IOM [38] and in hydroxy acids [39]. These enantiomeric excesses found in organic matter in chondrites may have been transferred to prebiotic molecules at the moment of the emergence of Life by various processes, including asymmetric catalysis [40]. This would have led to the appearance of homochirality, a strong property of terrestrial forms of Life. 


\section{The insoluble organic matter}

Elemental analysis of the IOM of Murchison IOM reveals typical formula to be $\mathrm{C}_{100} \mathrm{H}_{70} \mathrm{O}_{22} \mathrm{~N}_{3} \mathrm{~S}_{7}$. Two approaches can be used to study the molecular structure of the IOM: we can destroy its structure by thermal process (pyrolysis) or chemical reagents (mainly oxidants), or use spectroscopic techniques (like infrared spectrometry or solid state NMR). The molecular model [15] presented in figure 2 summarizes the results of 20 years of molecular analysis on Murchison IOM. Most of the carbon is involved in aromatic structures. Only 20 to $30 \%$ of the carbon constitutes aliphatic bonds. The maximum length is 7 carbon atoms for aliphatic bridges between aromatic units, and 4 carbon atoms for the side chains with a free end. Such carbon skeleton results in a high degree of crosslinking that may explain the chemical resistance of the IOM. It is also clearly different from typical terrestrial macromolecules found in sediments for instance, showing a different synthetic process.

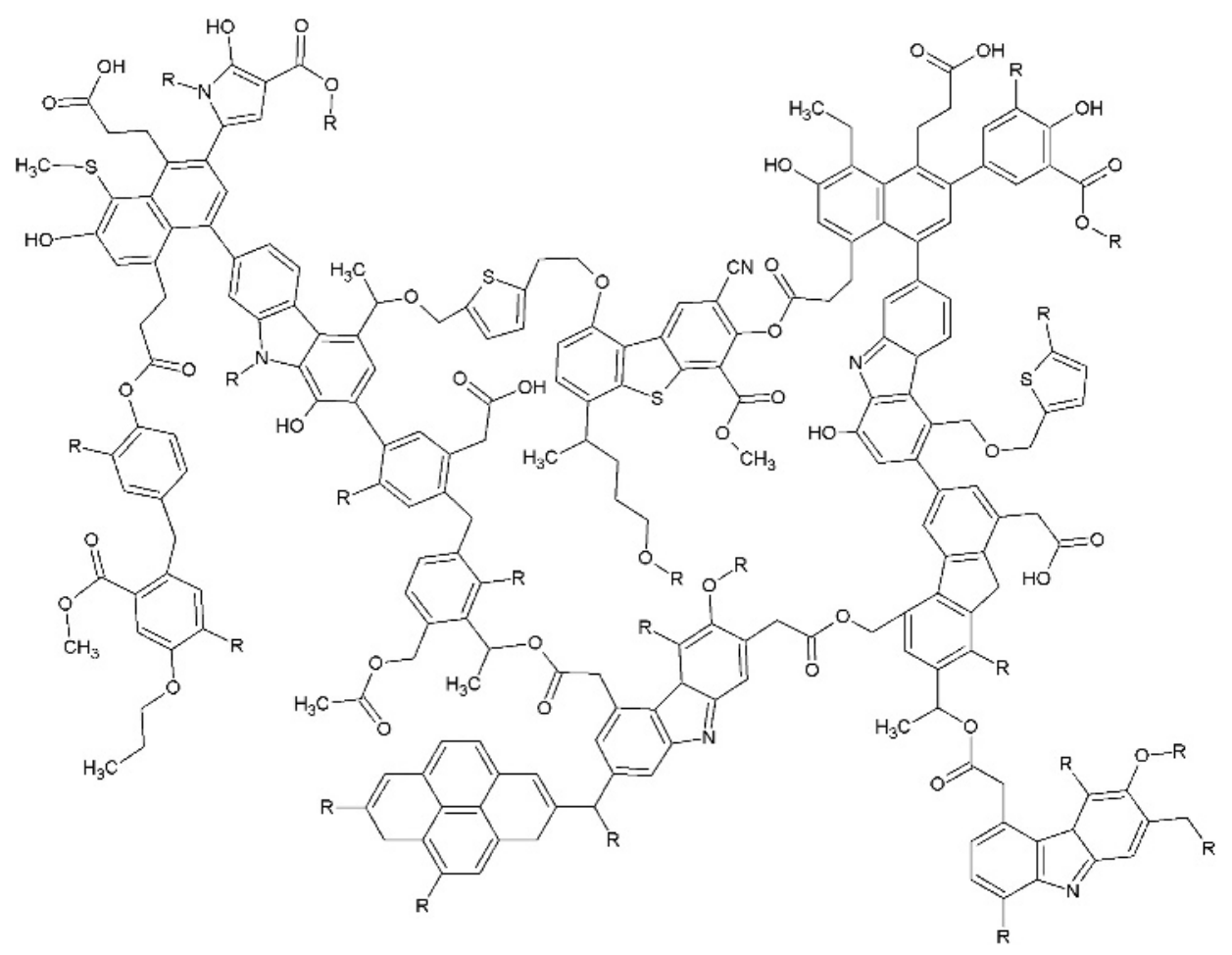

Fig. 2. summary of molecular information on the chemical structure of Murchison IOM. R means an organic moiety. From [15], modified.

The effects of parent body processes are noticeable. Thermal metamorphism induces dramatic modifications of the carbon skeleton through carbonization followed by graphitization. As shown by Raman spectrometry, the structural order of the carbonaceous matter increases with temperature [14]. There is a clear trend in losing the heteroelements, evidenced by a decrease in $\mathrm{H} / \mathrm{C}, \mathrm{N} / \mathrm{C}$ and $\mathrm{O} / \mathrm{C}$ elemental ratios, a loss of aliphatic chains and in increasing the size of aromatic units [41, 42]. On the other hand, hydrothermal alteration may induce oxidation of some functional groups like $\mathrm{O}$ and S containing groups. The S-containing groups have been shown to be indicators of the degree of alteration of the parent body [43]. Whereas S occurs as thiophenes (heterocycles) in less altered meteorites, like Murchison, more hydrated meteorites like Orgueil exhibit significant contribution of sulfoxide and sulfone in the aliphatic chains.

All these studies on chondrites indicate that the molecular structure of the macromolecule in carbonaceous chondrites can not be readily compared with models proposed for complex organic 
molecules in space (ISM or protosolar disks) based on observations [44] or laboratory experiments [45]. However, the carbon skeleton of less heated chondrites (CI, CM and CR) should not be significantly altered since the accretion of the parent body, as the molecular structure of their IOMs seems to be broadly similar. The question of the formation of the IOM in carbonaceous chondrites is still unresolved. Its structure has not yet been reproduced in laboratory and we still do not know if it is the result of photochemistry in ices, or chemistry in the gas phase or thermal processing of grains mantle. The chemical path may likely be a combination of all these processes, but the similarity in structure of the IOMs in various classes of chondrites imply a rather common process occurring in the parent molecular cloud or the protosolar nebula.

\section{The isotopic signature of water and organic matter in chondrites}

With the exception of presolar grains, characterised by isotopic compositions that can only be explained by nuclear processes in supernovae or evolved stars, most of chondrites components are thought to have formed during the early stages of the Solar System (in the so called protosolar nebula) or in the parent molecular cloud that evolved into our solar system. Despite numerous measurements, interpretations of the isotopic composition of organics and hydrated minerals in chondrites are still a matter of intense debate. It must be noted that in all the hydrated chondrites, water is always depleted in D compared to organic matter, whereas the equilibrium requires the opposite, whatever the location of the $\mathrm{H}$ in the molecule [46, 47]. This indicates that isotopic disequilibrium has been preserved during 4.56 billion years on the parent body of these carbonaceous chondrites and that parent body processes may not have erased the signatures of the synthesis.

\subsection{Water in chondrites}

In contrast to comets, meteorites do not contain any ice or liquid water. Water has reacted with minerals and produced phyllosilicates and other secondary phases in altered carbonaceous chondrites [48] on the parent body [11, 49] or during a pre-accretion process [50]. The water content in CM and CR goes from 3 to $14 \mathrm{wt} \%$, it goes up to $15 \mathrm{wt} \%$ in CI [51]. This water could have accreted as ice mixed with silicates grains or was trapped in phyllosilicates.

The $\mathrm{D} / \mathrm{H}$ ratio of water in chondrites is variable and may be influenced by parent body processes [51] and by pre accretion conditions, like temperature gradients in the protosolar nebula [52]. Meteoritic water has an average value around the terrestrial ratio, i.e. 6 times more enriched in D than the protosolar molecular hydrogen, but with some variations. In CI and CM, water is comprised within the range $-180 \%{ }_{0}<\delta \mathrm{D}<30 \%$ (i.e. $128 \times 10^{-6}<\mathrm{D} / \mathrm{H}<160 \times 10^{-6}$ ), but is heavier in $\mathrm{CR}$ chondrites: $\delta \mathrm{D}=680 \% ; \mathrm{D} / \mathrm{H}=262 \times 10^{-6}[53-55]$.

\section{2 $\mathrm{D} / \mathrm{H}$ ratio heterogeneities in the IOM}

Figure 3 shows the variations between the different classes of chondrites. In chondritic IOMs, $\delta D$ ranges from $-100 \%$ in enstatite chondrites up to $11000 \%$ in some ordinary chondrites [51]. CI and $\mathrm{CM}$, the most aqueously altered carbonaceous chondrites, exhibit similar ratios with the exception of Bells CM chondrite. CR chondrites, believed to be the less modified carbonaceous chondrites have a $\mathrm{D} / \mathrm{H}$ ratio twice as large; their IOM is often assumed to be the most pristine. $\mathrm{CO}$ and oxidized $\mathrm{CV}$, heated carbonaceous chondrites, have $\mathrm{D} / \mathrm{H}$ ratio around the terrestrial ratio; they are depleted compared to CI, CM and CR (2 times less compared to CM and 4 times less than CR). In these groups, the $\mathrm{D} / \mathrm{H}$ ratio does not correlate with an evolution in the $\mathrm{H} / \mathrm{C}$ ratio. Ordinary chondrites exhibit a negative trend in the diagram, with the samples with the lowest $\mathrm{H} / \mathrm{C}$ having the highest $\mathrm{D}$ enrichment. The reduced $\mathrm{CV}$ also exhibit the same trend, though the range of $\mathrm{D} / \mathrm{H}$ ratio is much smaller (a few hundred of permil compared to several thousands of permil in delta units). It must be 
noted that the lowest isotopic enrichment was measured in Abee enstatite chondrite $(\delta \mathrm{D}=-480 \%$; $\left.\mathrm{D} / \mathrm{H}=80 \times 10^{-6}\right)[56,57]$.

Isotopic imaging has revealed that $\mathrm{H}$ isotopes can exhibit large variations in the IOM of CI, CM and CR chondrites with the occurrence of D-rich " hot spots": micron sized areas with large positive $\mathrm{D}$ anomalies (figure 3), which could go up to $\delta \mathrm{D}=8000 \%$ o $\left(\mathrm{D} / \mathrm{H}=1402 \times 10^{-6}\right.$; [58-60]). No D-rich hot spots are observed in IOM of heated chondrites, i.e. CO, CV [60] pointing out to their thermal sensitivity. The distribution of the deuterium has also been studied at the molecular scale [61, 62]. A relation between the $\mathrm{C}-\mathrm{H}$ bond dissociation energy and the $\mathrm{D} / \mathrm{H}$ ratio has been revealed in Orgueil IOM [62]. In addition, radicals in the IOM of Orgueil are extremely D-rich: D/H $=15000 \pm$ $5000 \times 10^{-6} ; \delta \mathrm{D}=95300 \%$ [63]. Only these moieties have D-enrichment comparable to the highest enrichments observed in some organics in the ISM (45000 to $\left.60000 \times 10^{-6} ;[64]\right)$.

In summary, organics in chondrites are more D-rich than the protosolar $\mathrm{H}_{2}$, with $\mathrm{D} / \mathrm{H}$ ratios ranging from 5 to 20 times the protosolar $\mathrm{D} / \mathrm{H}$ (with a $\mathrm{D} / \mathrm{H}=25 \times 10^{-6}, \delta \mathrm{D}=-840 \%$, as determined from the Jupiter atmosphere and solar wind, [65]). Moreover the D is heterogeneously distributed between and within the samples, at the micron and the molecular scales. The interpretation of the $\mathrm{D} / \mathrm{H}$ ratio in the IOMs of carbonaceous chondrites remains very speculative: it has been attributed to interstellar [55, 57], nebular [62] or parent body processes [51]! This issue results from the superimposition of synthesis reactions (primary process) and the following modifications (secondary processes) that could affect the isotopic record of IOM in the gas phase or on the parent body.
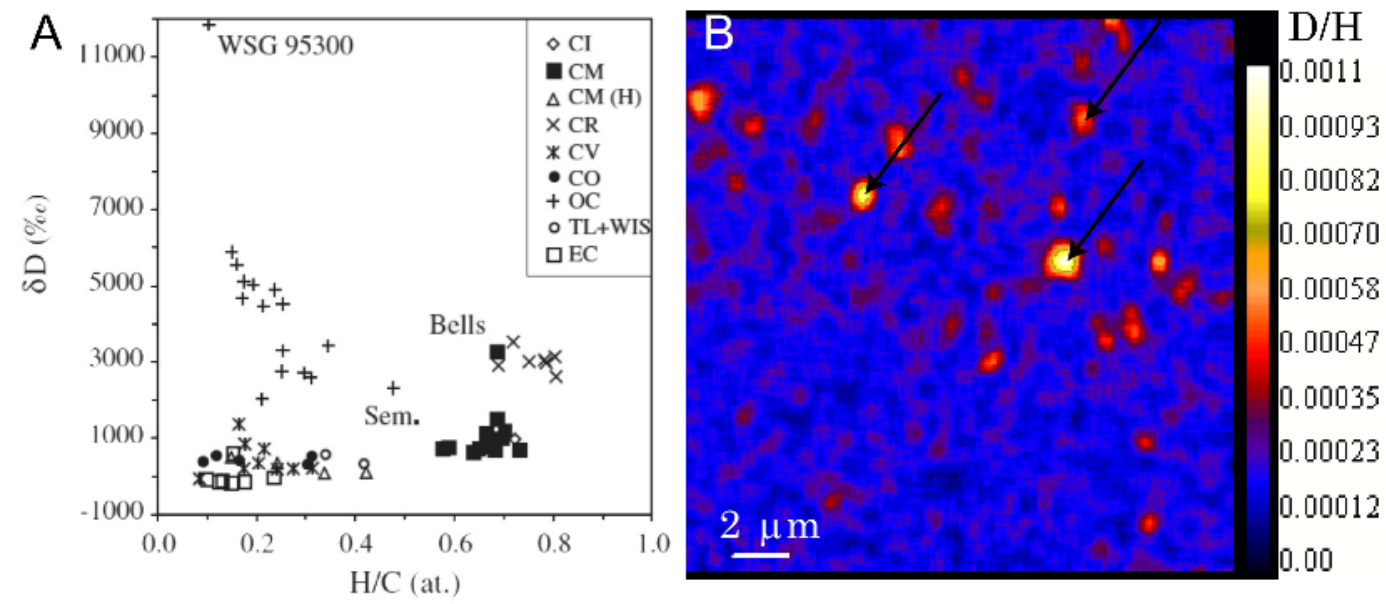

Fig. 3. Heterogeneous distribution of $\mathrm{D}$ in the IOM of chondrites. A: H isotopic compositions vs the $\mathrm{H} / \mathrm{C}$ ratio in the IOM of chondrites of various classes, from [51]. OC are ordinary chondrites, EC are enstatite chondrites, all the others are carbonaceous chondrites. B: NanoSIMS image of the IOM of the Orgueil meteorite, black arrows indicate exemples of D-rich hot spots. $\delta(\%)=($ Rsple $/$ Rstd -1$) * 1000$, with Rsple being the sample isotopic ratio and Rstd the ratio of a terrestrial standard : Standard Mean Ocean Water $\left(\mathrm{SMOW}: \mathrm{D} / \mathrm{H}=155.76 \times 10^{-6}\right)$ for $\mathrm{H}$ isotopes.

\subsection{Interpretation of the D content in organic matter and water}

The D-enrichment in chondritic organics in chondrites was interpreted as an interstellar origin, as large D-enrichments are predicted and observed in the ISM $[64,66]$. However, an interstellar origin of the $\mathrm{D} / \mathrm{H}$ ratio would require the precursor to survive and escape isotopic exchange during the ash conditions experienced in the ISM before the formation of the solar system or in the protosolar nebula during its early ages. From laboratory experiments [67], it has been shown that irradiations occurring in the ISM would likely erase a $\mathrm{D}$ rich signature and lower to $\mathrm{D} / \mathrm{H}$ ratio due to exchange with the molecular $\mathrm{H}_{2}$ gas (with a $\mathrm{D} / \mathrm{H}$ ratio close to $16 \times 10^{-6}$ ). Then, reactions during the formation 
of the solar system have to be considered to explain the D enrichment of the organics in meteorites. Two kinds of reactions could be involved in the synthesis of organics in chondrites: reactions at thermodynamic equilibrium between neutral species occurring in the disk mid plane, at relatively high temperature $(\mathrm{T}=400 \mathrm{~K},[68])$ and ion/molecule or gas/grain reactions occurring at lower temperature $(\mathrm{T}<100 \mathrm{~K})$ but under UV or X-ray irradiation [69]. Reactions involving ions or interactions between gas and grains are kinetically favoured at low temperature and are believed to induce large $\mathrm{D}$-enrichment in organic molecules $[66,70]$. In contrast, thermodynamic equilibrium in the warm midplane could theoretically induce only a modest D-enrichment in organic matter [71] with a $\mathrm{D} / \mathrm{H}<90 \times 10^{-6}$ in $\mathrm{CH}_{4}$, considering a starting $\mathrm{D} / \mathrm{H}$ of the protosolar nebula at $25 \times 10^{-6}$. These reactions would be kinetically inhibited [72] but catalytic processes on grain surfaces like FischerTropsch type reactions (FTT) have been proposed to produce a significant amount of organics under the assumed conditions of the inner protosolar disk [73, 74]. The $\mathrm{D} / \mathrm{H}$ of most chondrites IOMs, being much higher than $90 \times 10^{-6}$, can only be acquired in cold and ionized environments, by ion/molecule reactions for instance. Only the lowest $\mathrm{D} / \mathrm{H}$ could account for the occurrence of thermodynamic reactions.

The interpretations of the large heterogeneities in $\mathrm{D} / \mathrm{H}$ at different scales are also debated. The simplest hypothesis would be that a common organic precursor, with a $\mathrm{D} / \mathrm{H}$ signature related to its environment of synthesis, would have evolved under specific conditions of each parent body [51], leading to the variation of $\mathrm{D} / \mathrm{H}$ among the different classes as the result of isotopic exchange with fluids, loss of some organic reservoirs or reactions involving $\mathrm{H}$ transfer. In such a scenario, D-rich hot spots would represent remnant grains of interstellar D-rich organic material that would not be affected by parent body processes [58]. However, another hypothesis [62, 63] suggests that the Drich hot spots correspond to radical rich regions as organic radicals are D-rich and are heterogeneously distributed. As the radicals may have formed in the protosolar nebula, it would imply a local origin (i.e. not interstellar) for the D-rich hot spots. Then, if we can produce a large range of $\mathrm{D} / \mathrm{H}$ in organics within the protosolar nebula, or the parent molecular cloud, a pre-accretion diversity in isotopic composition of organic precursors could also explain the multiscale diversity in chondrites [46]. It must be noted that the most D-rich organic grains in chondrites does not show significant isotopic exchange with the mineral matrix, questioning a parent body effect to account for the observed diversity [46]. This assumption tackles the concept of a common precursor for all the chondrites and it implies that we should rather consider the organic matter in each chondrite group as a sampling of a range of isotopically and chemically distinct organic precursors. This diversity would reflect heterogeneities in the protosolar nebula or the parent molecular cloud: variations in ionization degree resulting in variations in the isotopic effect of ion/molecule reaction, different proportion of water/organics in ices depending on the location in respect to the snow line position, etc... Thanks to turbulence in the disk [75] organic particles could be exposed to very diverse environments and conditions before gathering at the location of the chondrites formations resulting in the association of very different organic grains in the same parent body.

\section{Conclusion}

Carbonaceous chondrites contain a diverse suite of molecules that could constitute the seeds of Life on Earth. Organic matter occurs in association with low temperature components in chondrites. The molecular characterization of the organic molecules in meteorites allows us to compare them with organic matter detected in the interstellar medium and in molecular clouds. This may fill the gap between the understanding of interstellar reactions and the extraterrestrial organic compounds brought to Earth by meteorites and comets and that were available in the Solar System before life started on Earth. Organic matter is characterized by a high and heterogeneous deuterium content. This heterogeneity cannot be fully explained by parent body effects and indicates that it is a mixing of components with several origins and history. Cold chemistry seems to be recorded in their $\mathrm{D} / \mathrm{H}$ ratio, but the physical conditions (location, temperature, irradiation, etc...) still needs to be 
constrained. This is required in order to build a self-consistent model that would explain the formation and the evolution of organics in the solar system.

However the influence of organic matter from carbonaceous chondrites on the emergence of Life remains debated and no laboratory experiment has proven it yet. It is nevertheless sure that the chondrites infall on the early Earth has released organic molecules in the prebiotic oceans. Interestingly, carbonaceous chondrites contain molecules able to produce spheres that could have induced the formation of the first cell membranes [76].

\section{References}

1. J.W. Truran Jr, A. Heger, D.H. Heinrich, and K.T. Karl, Treatise on Geochemistry, (Pergamon, Oxford, 2003).

2. Y. Amelin, A.N. Krot, I.D. Hutcheon, and A.A. Ulyanov, Sciencee 297, 1678 (2002).

3. $\quad$ E. Anders and N. Grevesse, Geochim. et Cosmochim. Acta 53, 197 (1989).

4. $\quad$ K. Lodders, Astroph. J. 591, 1220 (2003).

5. V.K. Pearson, et al., Met. Planet. Sci. 37, 1829 (2002).

6. B. Alpern and Y. Benkheiri, Earth Planet. Sci. Let. 19, 422 (1973).

7. L.A.J. Garvie and P.R. Buseck, Earth Planet. Sci. Let. 224, 431 (2004).

8. K. Nakamura, M.E. Zolensky, S. Tomita, S. Nakashima, and K. Tomeoka, Intern. J. Astrobio. 1, 179 (2002).

9. $\quad$ M. Saito and Y. Kimura, Astroph. J. 703, L147 (2009).

10. A.J. Brearley, D.H. Heinrich, and K.T. Karl, Treatise on Geochemistry, (Pergamon, Oxford, 2003).

11. T.E. Bunch and S. Chang, Geochim. et Cosmochim. Acta 44, 1543 (1980).

12. G.W. Kallemeyn and J.T. Wasson, Geochim. et Cosmochim. Acta 45, 1217 (1981).

13. M.K. Weisberg, T.J. McCoy, and A.N. Krot, Meteorites and the Early Solar System II, (University of Arizona Press, Tucson, USA, 2006).

14. L. Bonal, E. Quirico, M. Bourot-Denise, and G. Montagnac, Geochim. et Cosmochim. Acta Acta 70, 1849 (2006).

15. S. Derenne and F. Robert, Met. Planet. Sci. 45, 1461 (2010).

16. R.V. Krishnamurthy, S. Epstein, J.R. Cronin, S. Pizzarello, and G.U. Yuen, Geochim. et Cosmochim. Acta 56, 4045 (1992).

17. K.A. Kvenvolden, et al., Nature, 228, 923 (1970).

18. P. Schmitt-Kopplin, et al., Proc. Nation. Acad. Sci. 107, 2763 (2010).

19. G. Cooper, et al., Nature 414, 879 (2001).

20. Z. Martins, et al., Earth Planet. Sci. Let. 270, 130 (2008).

21. S. Pizzarello, G.W. Cooper, and G.J. Flynn, Meteorites and the Early Solar System II, (University of Arizona Press, Tucson, USA, 2006).

22. I. Gilmour, D.H. Heinrich, and K.T. Karl, Treatise on Geochemistry, (Pergamon, Oxford, 2003).

23. M.A. Sephton, C.T. Pillinger, and I. Gilmour, Geochim. et Cosmochim. Acta 62, 1821 (1998).

24. H. Yabuta, L.B. Williams, G.D. Cody, C.M.O.D. Alexander, and S. Pizzarello, Met. Planet. Sci. 42, 37 (2007).

25. J. Lawless, K.A. Kvenvolden, E. Peterson, C. Ponnamperuma, and E. Jarosewich, Nature 236, 66 (1972).

26. Z. Martins and M. Sephton, Amino Acids, Peptides and Proteins in Organic Chemistry. Vol.1 - Origins and Synthesis of Amino Acids, (Wiley VCH, Weinheim, 2009).

27. S. Pizzarello and W. Holmes, Geochim. et Cosmochim. Acta 73, 2150 (2009).

28. J.R. Cronin, G.W. Cooper, and S. Pizzarello, Adv. Sp. Res. 15, 91 (1995).

29. J.E. Elsila, S.B. Charnley, A.S. Burton, D.P. Glavin, and J.P. Dworkin, Met. Planet. Sci. 47, 1517 (2012). 
30. M.H. Engel and B. Nagy, Nature 296, 837 (1982).

31. D.P. Glavin and J.P. Dworkin, Proc. Nation. Acad. Sci. 106, 5487 (2009).

32. S. Pizzarello, M. Zolensky, and K.A. Turk, Geochim. et Cosmochim. Acta 67, 1589 (2003).

33. K. Soai, T. Shibata, H. Morioka, and K. Choji, Nature 378, 767 (1995).

34. E. Rubenstein, W.A. Bonner, H.P. Noyes, and G.S. Brown, Nature 306, 118 (1983).

35. U.J. Meierhenrich, et al., Angewandte Chemie-International Edition, 44, 5630 (2005).

36. W.L. Noorduin, et al., Nature Chemistry 1, 729 (2009).

37. Y. Takano, J. Takahashi, T. Kaneko, K. Marumo, and K. Kobayashi, Earth Planet. Sci. Let. 254, 106 (2007).

38. T. Kawasaki, et al., Geochim. et Cosmochim. Acta 70, 5395 (2006).

39. S. Pizzarello, Y. Wang, and G.M. Chaban, Geochim. et Cosmochim. Acta 74, 6206 (2010).

40. D.G. Blackmond, Proc. Nation. Acad. Sci. 101, 5732 (2004).

41. Y. Oba and H. Naraoka, Met. Planet. Sci., 44, 943(2009).

42. L. Remusat, C. Le Guillou, J.-N. Rouzaud, L. Binet, F. Robert and S. Derenne, Met. Planet. Sci. 43, 1099 (2008).

43. F.R. Orthous-Daunay, E. Quirico, L. Lemelle, P. Beck, V. deAndrade, A. Simionovici and S. Derenne, Earth Planet. Sci. Let. 300, 321 (2010).

44. Y.J. Pendleton and L.J. Allamandola, Astroph. J. Supplement Series, 138, 75 (2002).

45. E. Dartois, G.M. Muñoz Caro, D. Deboffle, G. Montagnac, and L. D'Hendecourt, Astron. Astrophys. 432, 895 (2005).

46. L. Remusat, Y. Guan, Y. Wang, and J.M. Eiler, Astroph. J. 713, 1048 (2010).

47. A.L. Sessions, S.P. Sylva, R.E. Summons, and J.M. Hayes, Geochim. et Cosmochim. Acta 68, 1545 (2004).

48. A.J. Brearley, Meteorites and the Early Solar System II, (University of Arizona Press, Tucson, USA, 2006).

49. E.R. DuFresne and E. Anders, Geochim. et Cosmochim. Acta 26, 1085 (1962).

50. A. Bischoff, Met. Planet. Sci. 33, 1113 (1998).

51. C.M.O.D. Alexander, S. D. Newsome, M. L. Fogel, L. R. Nittler, H. Busemann and G. D. Cody, Geochim. et Cosmochim. Acta 74, 4417 (2010).

52. F. Robert, Planet. and Sp. Sci. 50, 1227 (2002).

53. C.M.O.D. Alexander, R. Bowden, M. L. Fogel, K. T. Howard, C. D. K. Herd and L. R. Nittler, Science, 337, 721 (2012).

54. J.M. Eiler and N. Kitchen, Geochim. et Cosmochim. Acta, 68, 1385 (2004).

55. F. Robert and S. Epstein, Geochim. et Cosmochim. Acta, 46, 81 (1982).

56. L. Remusat, J. N. Rouzaud, E. Charon, C. Le Guillou, Y. Guan and J. M. Eiler, Geochim. et Cosmochim. Acta, 96, 319 (2012).

57. J. Yang and S. Epstein, Geochim. et Cosmochim. Acta, 47, 2199 (1983).

58. H. Busemann, A. F. Young, C. M. O. D. Alexander, P. Hoppe, S. Mukhopadhyay and L. R. Nittler, Science, 312, 727 (2006).

59. K. Nakamura-Messenger, S. Messenger, L.P. Keller, S.J. Clemett, and M.E. Zolensky, Science, 314, 1439 (2006).

60. L. Remusat, F. Robert, A. Meibom, S. Mostefaoui, O. Delpoux, L. Binet, D. Gourier and S. Derenne, Astroph. J., 698, 2087 (2009).

61. Y. Huang, M.R. Alexandre, and Y. Wang, Earth and Planetary Science Letters, 259, 517 (2007).

62. L. Remusat, F. Palhol, F. Robert, S. Derenne, and C. France-Lanord, Earth and Planetary Science Letters, 243, 15 (2006).

63. D. Gourier, et al., Geochim. et Cosmochim. Acta 72, 1914 (2008).

64. T.J. Millar, Solar system history from isotopic signatures of volatile elements, (ISSI, 2003).

65. J. Geiss and G. Gloecker, Sp. Sci. Rev. 84, 239 (1998).

66. S. Sandford, M.P. Bernstein, and J.P. Dworkin, Met. Planet. Sci. 36, 1117 (2001). 
67. G.M. Muñoz Caro, G. Matrajt, E. Dartois, M. Nuevo, L. D'Hendecourt, D. Deboffle, G. Montagnac, N. Chauvin, C. Boukari and D. Le Du, Astron. Astrophys. 459, 147 (2006).

68. B. Fegley, Sp. Sci. Rev. 90, 239 (1999).

69. A.J. Markwick, M. Ilgner, T.J. Millar, and T. Henning, Astron. Astrophys. 385, 632 (2002).

70. K. Willacy and P.M. Woods, Astroph. J., 703, 479 (2009).

71. C. Lécluse and F. Robert, Geochim. et Cosmochim. Acta 58, 2927 (1994).

72. R. Hayatsu and E. Anders, Top. Curr. Chem. 99, 1 (1981).

73. J.A. Nuth III, N.M. Johnson, and S. Manning, Astroph. J. 673, L225 (2008).

74. M.H. Studier, R. Hayatsu, asnd E. Anders, Geochim. et Cosmochim. Acta 36, 189 (1972).

75. F.J. Ciesla, Icarus 200, 655 (2009).

76. D. Deamer, J.P. Dworkin, S.A. Sandford, M.P. Bernstein, and L.J. Allamandola, Astrobiology 2, 792 (2002). 